\title{
Anabases
}

ANABASES Traditions et réceptions de l'Antiquité

$26 \mid 2017$

Varia

\section{La réception d'Homère dans le domaine de la rhétorique}

Notes de lecture à propos de l'ouvrage de S. Dubel, A.-M. Favreau-Linder et E. Oudot (éd.), À l'école d'Homère. La culture des orateurs et des sophistes, Paris (Éd. Rue d'Ulm de l'ENS), 2015

\section{Alexandra Trachsel}

\section{(2penEdition}

\section{Journals}

Édition électronique

URL : http://journals.openedition.org/anabases/6364

DOI : 10.4000/anabases.6364

ISSN : 2256-9421

\section{Éditeur}

E.R.A.S.M.E.

\section{Édition imprimée}

Date de publication : 1 novembre 2017

Pagination : 183-187

ISSN : 1774-4296

\section{Référence électronique}

Alexandra Trachsel, "La réception d'Homère dans le domaine de la rhétorique », Anabases [En ligne], 26 | 2017, mis en ligne le 01 novembre 2020, consulté le 20 janvier 2021. URL : http://

journals.openedition.org/anabases/6364; DOI : https://doi.org/10.4000/anabases.6364 


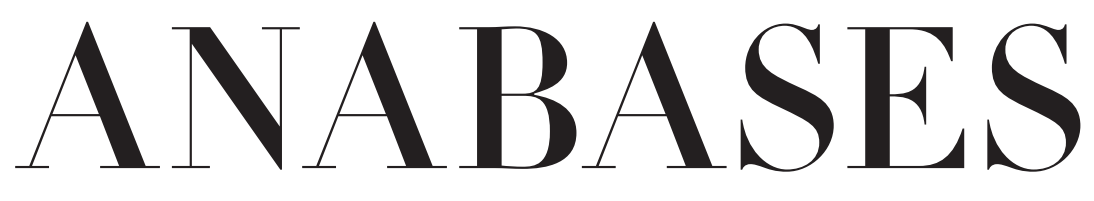

Traditions et Réceptions de l'Antiquité

$$
\begin{aligned}
& N \circ 26 \\
& 2017
\end{aligned}
$$

\section{E.R.A.S.M.E.}

Université Toulouse - Jean Jaurès 



\section{Sommaire}

$\mathrm{N}^{\circ} 26-2017$

\section{Historiographie et identités culturelles}

Jocelyne Peigney

La polysémie poétique d'anabase chez Saint-John Perse :

évocations, étymologie et botanique $\ldots \ldots \ldots \ldots \ldots \ldots \ldots \ldots \ldots \ldots$

Nicolas FAELLI

Les références antiques des fondateurs de l'Amérique française

au XVII siècle ................................. I9

Silvia Alaura

Setting the stage for Hittite studies in Victorian England:

practices and methods of the 1870 . . . . . . . . . . . . .

\section{Traditions du patrimoine antique}

“ Restituer Herculanum I. Des archives de fouilles aux restitutions 3D »

Alexandra Dardenay et Adeline Grand-Clément

Restituer Herculanum au XXI ${ }^{\mathrm{e}}$ siècle. L'apport des archives. . . . . . . . 55

Antonella Coralini

In situ e alibi, dallo scavo integrato alla cultura dell'abitare :

Vesuviana-DHER a Ercolano . . . . . . . . . . . . . . . . 67 67

Agnes Allroggen-Bedel

I documenti settecenteschi come strumenti per lo studio

degli scavi ercolanesi . . . . . . . . . . . . . . . Io3

James ANDREws

Revisiting the upper floors of the casa di Argo at Herculaneum . . . . . . II7 
Nicolas MonteIx

Les archives des fouilles d'Herculanum au XX $\mathrm{XX}^{\mathrm{e}}$ siècle :

analyse critique et pistes d'exploitation . . . . . . . . . . I43

\section{Archéologie des savoirs}

Yannick Le PAPE

L'inspiration et l'imprudence. Poésie de l'anticomanie

dans la critique d'art du second XIX ${ }^{\mathrm{e}}$ siècle . . . . . . . . . . . . . I57

\section{Actualités et débats}

Claude AzızA

Antiquités parallèles (7). La starlette des bandelettes . . . . . . . . . I77

Alexandra TrachseL

La réception d'Homère dans le domaine de la rhétorique $\ldots \ldots \ldots$. . . I83

Claude Aziza

Curiosa (2) In vino (vin/eau) veritas (very tasse) : drame de l'ivresse . . . . I89

\section{Lire, relire la bibliothèque des sciences de l'Antiquité}

Laurent OLIVIER

Pour en finir avec la race : Henri Hubert et l'anthroposociologie

À propos du commentaire de “L'Aryen, son rôle social », de Vacher de Lapouge $(\mathrm{r} 899) \ldots \ldots \ldots$

Henri Hubert

Compte rendu de Georges VACher de LAPouge, L'Aryen, son rôle social, L'Année sociologique, igoo, p. i45-146 . . . . . . . . . . . . . . . . . 203

\section{L'atelier de l'histoire : chantiers historiographiques}

L'Atelier des doctorants (coordonné par Adeline Grand-Clément) (14)

Francesco LoDÀ,

L'initiation des guerriers chez les peuples indo-européens.

Une enquête historiographique dans les pas de Georges Dumézil . . . 207

\section{Comptes rendus}

Benjamin Anderson et Robert G. Ousterhout

Palmyra I885. The Wolfe Expedition and the photographs

of John Henry Haynes (M. Sartre) . . . . . . . . . . . . . . . . . . . 2 215 
Mary R. BAchvarova

From Hittite to Homer. The Anatolian Background of Ancient Greek Epic (G. Hoffmann) . . . . . . . . . . . . . . . . .

Patrick Baker, Ronny Kaiser, Maike Priesterjahn, Johannes Helmrath (éd.) Portraying the Prince in the Renaissance. The Humanist Depiction of Rulers in Historiographical and Biographical Texts (G. Demelemestre). . 220

Brigitte BAKHоUсHE (éd.)

Science et exégèse. Les interprétations antiques et médiévales du récit biblique de la création des éléments (Genèse I, I-8) (F. P. BARONE) . . . . . . .

Laurence Baurain-Rebillard (éd.)

Héros grecs à travers le temps : autour de Persée, Thésée, Cadmos et Bellérophon (C. Giovénal) . . . . . . . . . . . . . . . .

Mark W. PAdiLla,

Classical myth in four films of Alfred Hitchcock . . . . . . . . . . . . . . .

Vivien Bessieres

Le Péplum et après? L’Antiquité gréco-romaine dans les récits contemporains (Cl. Aziza) . . . . . . . . . . . . . . . . . 225

Pierre Briant

Alexandre. Exégèse des lieux communs (É. Guillon) . . . . . . . . . . . . . 227

Paulo ButTi DE Lima

Il piacere delle Immagini. Un tema aristotelico nella riflessione moderna sull'arte (C. Noacco) . . . . . . . . . . . . . . . . . . .

Bénédicte Coudì̀re

La truelle et le phylactère. La proximité des images (M. Scapin) . . . . . . . 23I

Thorsten Fögen, Richard WARREN (éd.)

Graeco-Roman Antiquity and the Idea of Nationalism in the rgth Century, Case Studies (M. Champier) . . . . . . . . . . . . . . . ${ }_{232}$

Ronald H. Fritze

Egyptomania. A History of Fascination, Obsession and Fantasy

(Fabien Bièvre-Perrin) . . . . . . . . . . . . . . . . . . . . . 234

Joseph Geiger

Hellenism in the East. Studies on Greek Intellectuals in Palestine

(C. Bonnet). . . . . . . . . . . . . . . . . 236

Tiffany Jenkins

Keeping their marbles. How the treasures of the past ended up in museums... and why they should stay there (C. Valenti) . . . . . . . . . . 238 
Ayelet H. Lushkov

Magistracy and the Historiography of the Roman Republic.

Politics in Prose (C. Landrea) . . . . . . . . . . . . . . . . . . . . . . 240

Jean-Claude MüHLEthaler

Énée le mal-aimé. Du roman médiéval à la bande dessinée

(Fl. Bouchet) ........................ 24I

Laurent PERNoT

Epideictic Rhetoric, Questioning the Stakes of Ancient Praise

(C.Psilakis) ......................... 243

Priscian

Answers to King Khosroes of Persia (O. Gengler) . . . . . . . . . . . . . . 245

Paul Robertson

Paul's letters and contemporary Greco-Roman literature:

theorizing a new taxonomy (M. Cambron-Goulet) . . . . . . . . . . . . . . 247

Federico Santangelo (éd.)

Approaching the Roman Revolution. Sir Ronald Syme,

Papers on Republican History (C. Landrea) . . . . . . . . . . . . . . . . . . 249

Molly Swetnam-BurLand

Egypt in Italy. Visions of Egypt in Roman Imperial Culture

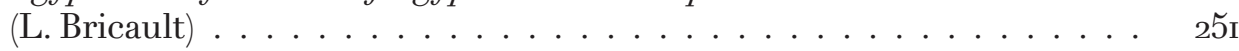

Helen VAN Noorden

Playing Hesiod. The 'Myth of the Races' in Classical Antiquity

(D. Bonanno)

Résumés . . . . . . . . . . . . . . . . . . . . . . . . . . . . . . . 257

Index .......................... . . 265 


\section{Actualités et débats}





\section{La réception d'Homère dans le domaine de la rhétorique}

Notes de lecture à propos de l'ouvrage de S. Dubel, A.-M. Favreau-Linder et E. Oudot (éd.), À l'école d'Homère. La culture des orateurs et des sophistes, Paris (Éd. Rue d'Ulm de l'ENS), 2015

Alexandra Trachsel

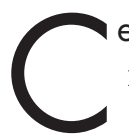

e volume comprend les actes d'un colloque à deux volets consacré à la réception d'Homère dans le domaine de la rhétorique. La réception d'Homère, étudiée abondamment dans d'autres domaines, n'avait pas encore fait l'objet d'une étude de synthèse dans le champ de la théorie de la rhétorique ancienne. La réussite de l'entreprise nous paraît incontestable et nous fait attendre avec impatience le second volume annoncé. L'ouvrage se divise en quatre parties, précédées d'un texte programmatique de F. Kimmel-Clauzet (Homère, le premier des sophistes ?), consacré à deux des vies d'Homère conservées : la Vie du ps.-Hérodote et la Dispute d'Homère et d'Hésiode. L'A. montre qu'au moment où celles-ci ont été rédigées, il était possible de considérer le personnage d'Homère comme un sophiste; avec ce constat, est annoncé le thème de tout l'ouvrage.

La première partie, Grecs et Romains à l'école d'Homère, réunit cinq contributions qui démontrent, chacune à sa façon, comment les orateurs, à partir du $\mathrm{V}^{\mathrm{e}} \mathrm{s}$. av. J.-C., se sont inspirés des deux poèmes homériques, souvent pour en récrire certains épisodes dans un contexte nouveau. R. Ahern Knudsen (Homer in the First Sophistic. A Study of Four Speeches) discute quatre exemples tirés de textes de sophistes du V ${ }^{\mathrm{e}} \mathrm{s}$. L'A. analyse ceux des discours de Gorgias, d'Antisthène et d'Alcidamas qui revalorisent, souvent dans un but pédagogique, certains héros 
homériques (Palamède, Ulysse et Ajax), pour les présenter sous un nouveau jour. D. van MaL-Maeder (Testis carminum antiquitas. Homère dans la rhétorique et les déclamations latines) explore la présence d'Homère dans les déclamations latines et la rhétorique judiciaire romaine. L’A. constate que, malgré la mention chez les théoriciens de la rhétorique latine d'exemples homériques à suivre, au niveau du contenu ou du style, l'utilisation dans la pratique est plutôt rare et reste voilée. Elle invoque le contexte culturel romain et la question de la diffusion de la langue grecque pour expliquer ce constat et pour décrire cette forme latine de la réception. P. Cauderlier (Deux parerga homerica [ I $^{\text {er }}$ et II ${ }^{e}$ s.] ou l'utilisation des textes homériques par le rhétorikos et le sophistès dans la province d'Égypte) étudie deux exemples de réception homérique conservés sur papyrus (P. Oxy. 221 et P. Lond. III 734). Le premier témoigne de l'étendue de la philologie homérique et le second préserve une interprétation allégorique proche de celles que nous trouvons dans certains traités de Plutarque. Les deux textes montrent l'influence que les poèmes homériques exerçaient en Égypte à l'époque impériale, sous forme d'exégèses allégoriques. F. Roвert (La présence d'Homère dans les Progymnasmata d'époque impériale) s'attache aux Progymnasmata, dont les exemplaires conservés ont été rédigés entre le $\mathrm{II}^{\mathrm{e}}$ et le $\mathrm{V}^{\mathrm{e}} \mathrm{s}$. ap. J.-C. Il montre que dans ces traités les poèmes homériques restent des sources d'inspiration et fournissent des exercices aux élèves, mais ne sont plus compris comme des modèles à suivre au niveau du style. La contribution de B. Schouler (Pour les sophistes, Achille ne fut-il que colère?) illustre le traitement que l'on réservait à Homère dans l'Antiquité tardive, en nous montrant l'attitude de Libanios à l'égard d'Homère, particulièrement à propos d'Achille. Le rhéteur prend une certaine liberté face au modèle homérique et ajoute plusieurs thématiques nouvelles au traitement du caractère d'Achille. Les contributions de cette première partie montrent toutes comment Homère reste la référence culturelle commune à travers les siècles comme source d'inspiration. Elles témoignent aussi d'un progressif éloignement du modèle, notamment au niveau du style, et c'est cet aspect qui devient le fil conducteur de la deuxième partie (Stratégies rhétoriques : modèles et détournements).

J. Peigney (La guerre de Troie dans le discours d'Ulysse à Polyphème chez Euripide [Cyclope, 285-312]) montre que les discours d'Ulysse et de Polyphème dans le Cyclope d'Euripide s'adaptent à un contexte athénien, où les personnages font allusion aux enjeux soulevés par les guerres médiques ou la guerre du Péloponnèse. M. Tamiolaki (Homère chez Isocrate: source de rivalité ou d'inspiration?) décrit l'attitude ambiguë d'Isocrate à l'égard d'Homère. L'orateur athénien n'adopte pas le point de vue très critique d'un Platon, mais veut tout de même démontrer la supériorité de son propre art oratoire. Tout en louant le génie du grand poète, Isocrate transforme et adapte à sa guise les éléments empruntés aux textes homériques. D. KasprzyK (Homère travesti. À propos de Dion, Discours 
aux Alexandrins [Or. XXXII], 82-85) discute un détournement particulier, prenant comme objet d'étude le centon homérique que Dion de Pruse insère dans son discours aux Alexandrins pour critiquer leur comportement inapproprié lors des spectacles. Comme il s'agit d'une recomposition faite à partir de vers homériques, le texte du modèle est présent à chaque ligne. Pourtant, comme les vers sont décontextualisés, puis recontextualisés, ils prennent un autre sens et permettent à Dion de créer son propre message, entièrement nouveau est adapté à la situation d'énonciation actuelle. L. MiLetri montre comment Homère sert aussi de point de départ dans le cas de l'éloge de soi-même (Homère comme modèle pour l'éloge de soi-même. Autour du discours XXVIII Keil d'Aélius Aristide). Dans ce contexte, l'A. analyse l'exemple d'Aélius, mais le situe dans une tradition plus longue d'auteurs qui se sont prononcés sur ce sujet, assez délicat, puisqu'il va à l'encontre de la modération attendue dans un discours. Pour justifier l'exercice, on se référait à des exemples homériques où un tel éloge de soi-même était admis (Nestor et Ulysse). C'est aussi ce que fait Aélius : il se prétend inspiré par Asclépios et ajoute aux exemples d'Ulysse et de Nestor le passage de l'hymne homérique à Apollon où le poète s'adresse à un chœur de jeunes filles pour louer la renommée de son propre art. M. BRIAND (L'Homère paradoxal de Lucien. Un dialogue, entre imitation et satire) analyse l'ironie de Lucien par rapport au modèle homérique. Le dialogue entre Lucien et Homère s'instaure à plusieurs niveaux dans l'œuvre de Lucien, mais de manière particulière dans les Histoires vraies. Mettant en scène un Homère désinvolte et lui-même critique face à la philologie homérique, Lucien le tire dans son camp et lui accorde une place privilégiée qui lui permet de le louer malgré le détachement exigé par l'ironie. Cette section montre ainsi que, malgré la grande liberté que les orateurs prennent par rapport au modèle homérique, demeure une certaine révérence.

La troisième partie, Enjeux critiques, développe un autre aspect de la thématique. S. Gotteland (Homère dans les écrits socratiques de Xénophon) traite de Xénophon qui nous propose un regard différent, par rapport à Platon, sur l'attitude de Socrate envers Homère, en mettant en scène les premiers exégètes homériques. Pour le Socrate de Xénophon cette exégèse d'Homère doit aller audelà du simple apprentissage par coeur de vers homériques. Pour lui, le travail sur Homère devrait être le point de départ d'interprétations plus approfondies et de réflexions personnelles. Une attitude qui permet à l'A. de constater qu'à partir de Xénophon l'exégèse du texte homérique peut également devenir l'objet d'un discours ou d'une réflexion. K. Oiкonomopoulou traite de Plutarque et de son rapport à Homère et à la critique littéraire (Rhetoricians on Homer. The Antinomikon Zètèma of Plutarch's Quaestiones Convivales 9.13). L'A. se concentre en particulier sur la discussion d'un passage du chant 3 de l'Tliade où il est question des serments que s'échangent Achéens et Troyens. Ces vers permettent à Plutarque de décrire 
le savoir des rhéteurs de son temps et à l'A. de monter comment Homère et toute la philologie qui s'était créée autour de ses textes étaient utilisés dans les milieux cultivés. Enfin, R. Wевв (Homère et les Images de Philostrate) analyse le rapport que Philostrate établit, dans certaines de ses descriptions à sujets homériques, entre les images décrites et le texte homérique. L’A. montre que ces descriptions sont aussi des sortes de commentaires autant en ce qui concerne la technique narrative (opposant les images, basées sur une perception immédiate du thème illustré, aux récits dans lesquels celui-ci est présenté) que les thématiques (scènes liées à Antiloque, Cassandre et le Scamandre).

La dernière partie enfin, intitulée Héritages, est consacrée à l'Antiquité tardive et à l'époque byzantine. A. Pizzone (Thersite au bord du Nil. Homère et l'imaginaire des “wandering sophists» de Gaza) nous présente Énée de Gaza, un auteur peu connu, qui illustre comment Homère était lu au VI ${ }^{\mathrm{e}}$ s. apr. J.-C. C'est par un souvenir de jeunesse concernant une performance rhétorique à Alexandrie qu'Enée revient à Homère. Lui-même et le destinataire de sa lettre avaient jadis comparé Thersite, le plus laid des personnages homériques et Nirée le plus beau des hommes. En évoquant ce souvenir, Énée résume les interprétations diverses que les érudits avaient faites du personnage et témoigne par là d'une attitude ludique face à Homère, ce qui le distingue de l'interprétation chrétienne. A. Sтовнr-Monsou (Une réception rhétorique d'Homère en Afrique vandale: Dracontius [Romulea, VIII-IX]) présente un exemple emprunté au domaine latin en parlant de deux poèmes de Dracontius. Celui-ci veut renouveler la tradition tout en rendant hommage au poète. Il réalise le second but dans le Rapt d'Hélène, où Dracontius rend hommage à Homère et à Virgile en les invoquant au début du poème. En revanche, dans la Romulea IX il rivalise avec Homère en récrivant l'épisode de l'ambassade de Priam auprès d'Achille. Il témoigne aussi de toute la tradition rhétorique développée autour de cette scène, se montrant un homme de lettre éduqué, en milieu latin. D. Pralon (Les personnages d'Homère selon Isaac Comnène Porphyrogénète. Les portraits d'Agamemnon et de Ménélas) aborde le milieu byzantin, avec Isaac Comnène $\left(\mathrm{XI}^{\mathrm{e}}-\mathrm{XII}^{\mathrm{e}} \mathrm{s}\right.$.) et sa description des caractères des personnages de l'Iliade. Son entreprise, qui n'apporte, selon l'A., que peu de nouveauté, si ce n'est qu'il s'agit de la première systématisation de portraits textuels, témoigne d'un renouveau d'intérêt pour les deux poèmes homériques. C'est au Byzantin Eustathe et à deux de ses contemporains, que M. LoukaKI, consacre sa contribution (L'univers homérique dans les éloges impériaux du XII $s$. à Byzance. Notes sur Théophylacte d'Achrida, Nicéphore Basilakès et Eustathe de Thessalonique). Ces trois érudits ont ceci en commun d'avoirécrit des panégyriques impériaux dans lesquels ils ont introduit des réminiscences homériques. Cellesci se manifestent principalement par des citations de vers homériques, même si Eustathe fait aussi allusion aux exégèses avancées dans ses propres commentaires. Cependant, les trois érudits demeurent très loin du monde homérique. 
Il faut souligner la richesse de ce recueil qui explore une grande variété de lectures et de réécritures de la tradition homérique dans le domaine de la rhétorique antique. Les contributions montrent qu'Homère servait de modèle et d'inspiration aux orateurs, puisqu'il était perçu comme le premier des sophistes, qui possédait des talents oratoires exceptionnels dont on pouvait ou devait s’inspirer. Il était aussi un modèle avec lequel on pouvait rivaliser, en réinventant les fameux épisodes de ses poèmes ou en les adaptant à ses propres principes rhétoriques. On y voit aussi que la mise en scène de spécialistes de l'exégèse homérique pouvait servir à montrer l'attachement qu'un auteur avait à l'égard de la tradition ou lui permettait de définir la distance qu'il voulait établir par rapport au grand modèle. La réception d'Homère chez les rhéteurs a donc été très féconde et a produit un grand nombre de réponses qui permettaient à chacun de montrer ses talents. Le lecteur apprendra dans cet ouvrage tout autant sur les poèmes homériques et leur réception que sur le regard que les rhéteurs, latins ou grecs, portaient sur leur métier à travers les âges.

\author{
Alexandra Trachsel \\ Université de Hambourg \\ von-Melle-Park 6 \\ 20146 Hamburg \\ alexandra.trachsel@uni-hamburg.de
}


